\title{
Germanica
}

\section{Literatur des Vergessens Brechts Strategien für Städtebewohner und die Kritik der Erinnerung}

Littérature de l'oubli. Stratégies urbaines selon Brecht et la critique de la mémoire

\section{Franck HOFMANN}

\section{(2)enEdition}

\section{Journals}

Édition électronique

URL : http://journals.openedition.org/germanica/1825

DOI : 10.4000/germanica. 1825

ISSN : 2107-0784

\section{Éditeur}

Université de Lille

\section{Édition imprimée}

Date de publication : 31 décembre 2003

Pagination : 79-96

ISBN : 9782913857124

ISSN : 0984-2632

\section{Référence électronique}

Franck HOFMANN, «Literatur des Vergessens Brechts Strategien für Städtebewohner und die Kritik der Erinnerung », Germanica [Online], 33 | 2003, Online erschienen am: 03 Dezember 2012, abgerufen am 06 Oktober 2020. URL : http://journals.openedition.org/germanica/1825 ; DOI : https://doi.org/ 10.4000/germanica.1825

Ce document a été généré automatiquement le 6 octobre 2020.

(c) Tous droits réservés 


\section{Literatur des Vergessens Brechts Strategien für Städtebewohner und die Kritik der Erinnerung}

Littérature de l'oubli. Stratégies urbaines selon Brecht et la critique de la mémoire

Franck HOFMANN

Lobet von Herzen das schlechte Gedächtnis des

Himmels !

Und daß er nicht

Weiß euren Nam noch Gesicht Niemand weiß, daß ihr noch da seid.

Bertolt Brecht, Der Große

Dankchoral.

\section{Brechts urbane Literatur im Kontext der ARS OBLIVIONIS}

Eine Literatur des Vergessens kann in Bertolt Brechts Arbeiten aus den ersten Jahrzehnten des letzten Jahrhunderts ein prominentes Beispiel finden. Mit der Suche nach neuen Lebensstrategien für Städtebewohner wird auch die Kritik der Erinnerung zum Thema insbesondere in Gedichtsammlungen wie «Hauspostille» (1927) und «Lesebuch für Städtebewohner»(1930), in Dramen wie etwa «Im Dickicht der Städte ", das 1927 als zweite Fassung des bereits vier Jahre zuvor bearbeiten Stoffes erscheint. Die Frage nach Stellenwert, Funktion und angemessener Fassung des Gedächtnisses wird nicht nur in philosophischen und psychologischen Überlegungen, wie etwa 1896 in der für die deutschsprachige Lebensphilosophie und Phänomenologie des ersten Jahrhundertdrittels wirksamen Schrift Bergsons über «Matière et Mémoire » oder in Freuds « Notiz über den Wunderblock» von 1925 diskutiert : Sie ist gerade auch prominenter Gegenstand einer Literatur, die, als Teil der ARS OBLIVIONIS verstanden, selbst zu einer Verlaufsform der Erinnerung und zur Praxis eines sich 
erneuernden urbanen Menschen gerät. So kann etwa Brechts urbane Literatur des Vergessens phänomenologischer und psychoanalytischer Gedächtniskritik als eine eigenständige literarische Position zur Seite gestellt werden. Ihre Texte fragen nach Funktion und Verfassung des kulturellen ebenso wie des individuellen Gedächtnisses, sie thematisieren retrograde Bindungen der europäischen Kulturen im Zeichen des Urbanen ebenso wie Generationenbezüge als Teil eines umfassenden Verständigungsproblems der vom "Einzug der Menschheit in die großen Städte » erfüllten Zeit. (Brecht: Werke Bd. 21, 113) ${ }^{1}$. Auf die von Brecht hier als Herausforderung einer neuen Dramatik gestellte Diagnose reagiert er mit der Bemühung um eine urbane Sprache, die ihren Gegenständen wie ihren Formen und Praktiken nach gerade auch auf einer Umarbeitung der Erinnerung beruht. Deren angemessene literarische Fassung ist Teil der Suche nach einer neuen Sprache der großen Städte, in denen mit dem bürgerlichen Menschen nicht zuletzt dessen Gedächtnis und ein Identität stiftender, retrograder Bildungszusammenhang unter Druck geraten sind. Ist in den Metropolen doch nicht nur der Generationszusammenhang sondern auch ein Kulturmodell brüchig geworden, in dem die Konzeption des Subjekts retrograd im Erbe des europäischen Humanismus begründet war : Die Antike taugt nicht mehr als Vorbildkultur unter den Verhältnissen eines wissenschaftlich - technischen Fortschritts, der das Leben in den Städten in einer neuen Dimension prägt und von Brecht auch zum Ausgangspunkt der von ihm anvisierten Erneuerung der Sprache gewählt wird, die darauf verzichtet, den brüchig gewordenen humanistischen Grund urbanisierter Gesellschaften zu stabilisieren, wie es etwa die idealistischen Reprisen im deutschsprachigen Expressionismus noch anstrebten (Vgl.: Brecht: Werke 21, 168). Im Gegenteil. Die Neuausrichtung der Erinnerung im Rahmen der ARS OBLIVIONIS wird aus symbolphilosophischer Perspektive insbesondere an der Bruchkante zu einem humanistischem Erbe des Mittelmeerraums diskutiert. Dieses verliert spätestens um 1900 seine Legitimationsmacht und wird Gegenstand neuer Versuche der Traditionsbestimmung in den Kunst- und Literaturwissenschaften ${ }^{2}$. Doch ebenso wie diese reagieren auch die Literaturen selbst auf den als Form- und Sprachkrise evident werdenden Bruch. Sie antworten auf die Einsicht, dass tradierte Begriffe der Kunst und ihre Allegorien nicht mehr tauglich sind, eine neue Qualität urbaner Wirklichkeit zu erfassen und in diese mit Hilfe ästhetischer Praktiken zu intervenieren. - Sei es, indem sie - wie Brecht - den gebrochenen retrograden Bezug noch verschärfen und den Abstand in allen Bereichen eines urbanen Lebens und seiner Sprache inszenieren, sei es, indem sie - wie etwa Rudolf Borchardt den schwindenden Bezug zum Gegenstand einer literarischen Einbildungskraft machen und in ihrer Dichtung als eine Erfindung wieder zu etablieren suchen ${ }^{3}$. Reagieren die einen, die Vertreter der literarischen Neuklassik und -Romantik um 1900, etwa auf die Krise des philosophischen Idealismus mit einer Metaphysik der Kunst, plädieren andere in ihrer Antwort auch auf solche neuhumanistischen Bemühungen für eine radikale Kritik des idealistischen und metaphysischen Erbes. Diese treiben sie in der Umkehrung der es formierenden Kategorien, Praktiken und Werte voran : etwa auch der - nicht mehr - Sinn stiftenden Erinnerung. Beiden Strategien scheint jedoch eine wenn auch unterschiedlich ausgeprägte - Aufmerksamkeit für das symbolische Vermögen der Formen und der zu erneuernden Sprache gemeinsam. Der Arbeit an der Sprache werden auch die Gegenstände der Erinnerung unterstellt, die mit den retrograden Traditionsbezügen des Menschen unter Druck geraten ist und in der literarischen Praxis weniger ihren Inhalten oder ihrer medialen Verfassungen nach, 
sondern mit Blick auf eine Praxis der Formen bearbeitet wird. Die literarisch entfaltete Kritik der Erinnerung ist so verstanden Teil einer Umwertung des humanistischen Erbes und antwortet auf dessen Entwertung im Übergang zu einem Leben in den großen Städten, das sich auch die Bilder der Natur und der Mythen unterstellt, zu einem Leben, das mit jeglicher Transzendenz auch die retrograde Bindung in seine spezifische Gegenwärtigkeit einzieht. Ein als Teil der ARS OBLIVIONIS entworfenes Lob des Vergessens richtet sich gegen den Vorrang eines überbordenden Gedächtnisses, das auch Teil eines ausgreifenden Positivismus des Wissens ist, das den Platz einer antiquarischen Geschichte einnimmt. In der Nachfolge Nietzsches gilt seine Kritik in den ersten Jahrzehnten des 20. Jahrhunderts einem akkumulierten Wissen und einem auf dieses gründenden, retrograd gebundenen Fortschrittsmodell, in dem eine lebendiger Verbindung zur Tradition durch abstrakte Erinnerungsbezüge ersetzt scheint. - Dies geschieht um den Preis, dass deren literarische Dimension vielfach unverstanden bleibt oder zumindest nicht explizit herausgestellt wird. Ohne in eine unmittelbare Materialität zurückzufallen - die in der Sprache eines ekstatischen, die Sinne auszeichnenden Vitalismus nur noch zitiert wird - zielt das Lob des Vergessens letztlich auf eine Umkehrung des dominierenden, auf abstrakte Erinnerung, ihre Medien und Zeitmodelle basierenden Zivilisationsmodells.

\section{Rimbaud als Gewährsmann einer Literatur des Vergessens}

2 Stärker als von Nietzsches Kritik der Geschichtsphilosophie, deren Lektürespuren in der Generation Brechts allgegenwärtig sind und die Bruchstellen mit der Generation der literarischen und philologischen Gewährsmänner markiert, scheint diese urbane Literatur des Vergessens allerdings von Rimbauds Plädoyer für eine um den Sehsinn zentrierte materialistische Dichtung beeinflusst ${ }^{4}$. Dessen 1928 veröffentlichte «lettres du voyant" und die Verse des "bateau ivre" von 1871 stehen so - bis in die Gegenstände und Bildwelten Brechts hinein spürbar - im Hintergrund einer ästhetischen Praxis, die ein neues Verhältnis von Erinnerung und Fortschritt im Zeichen der Urbanen als Frage nach der Sprache und der Lebensfähigkeit des Menschen entwirft. Nicht zufällig wird Brechts «Im Dickicht der Städte » mit einem Hinweis auf Rimbaud eröffnet und mit einer Kritik der Würdeformen der Erinnerung verbunden. Der Kampf zweier Männer, dem die Aufmerksamkeit des Zuschauers gelten soll, setzt mit einem Abstand zur Buchkultur ein, der zugleich mit der ersten Verunsicherung des aus der Provinz zugezogenen George Garga hergestellt wird : «Bücher! Wozu helfen sie? Wurde das Erdbeben von San Francisco aufgehalten durch Bibliotheken?» (Brecht: Werke 1, 442). Er könne seinem Gegner, so der hier angesprochene, in einer Leihbibliothek arbeitende Garga, die Ansichten von Jensen oder Rimbaud verkaufen, jedoch nicht - wie von diesem gefordert - seine Ansichten zu diesen. (Ebd., 439). Neben den genannten Autoren sind drei weitere Traditionen aufgerufen, in deren Spur Brechts urbane Literatur zu lesen ist: Kriminalroman und Reiseliteratur werden von Garga empfohlen. Mit diesen wird auch auf das Lexikon verwiesen : von Dichtung und idealistischer Erbauung an der Überlieferung keine Spur. Garga hat vielmehr «die Gewohnheit einige Wochen gleichzeitig zu trinken, zu lieben und zu rauchen. »- Und eben, wie Shlink ergänzt : «[D]as Konversationslexikon durchzublättern. » (Ebd., 447) Am Ende des Kampfes, wenn Garga sein « rohes Fleisch in die Eisregen » hinaus tragen 
wird (Ebd., 494), ist auch dieser Bezug auf die retrograde Buchkultur noch zugunsten eines gegenwärtigen Vitalismus getilgt, der einer Auslöschung des Alten verbunden ist :

Garga : ‘...] Ich werde schwimmen, Gras zerstampfen, jagen, rauchen vor allem. Getränke trinken wie kochendes Metall. Ich werde mich ins Leben mengen, gerettet sein.' - Was für Dummheiten! Worte, auf einem Planeten, der nicht in der Mitte ist ! Wenn sie längst Kalk über sich haben, durch die natürliche Ausscheidung des Veralteten, werde ich wählen, was mich unterhält. (Ebd., 492-493)

Brecht, der hier aus Adolf Christians Übertragung von Rimbauds «Une saison en enfer» zitiert (Vgl.: Ebd., 605-606), markiert zugleich die Distanz, in der seine Verarbeitung zu Rimbauds Vitalismus steht : die Reichweite der Worte ist angesichts der mondialen Herausforderung durch das Urbane begrenzt. Für Rimbaud interessiert sich Brecht gleichwohl nicht zuletzt auch wegen des hochfahrend - jugendlichen Habitus und des die Gestalt des Autor umlagernden Mythos des Reisenden. Rimbauds Programm, auf das Brecht reagiert, wird insbesondere auch in den " lettres du voyant " deutlich, in denen dieser ebenfalls auf den notwendigen Bruch der (kulturellen) Genealogie setzt. Statt in dieser verhaftet zu sein, sei es, so Rimbaud, für einen an sich arbeitenden Menschen wichtig, in der Fülle des Traums zu leben. Ein Mensch, der Dichter werden wolle, müsse sich kennen, sich bilden und darauf hin streben, seine Seele ungeheuerlich zu machen. (Vgl. Rimbaud: Lettres du voyant, 24). In der von Brecht vorgeschlagenen Ausbildung Gargas zum Städtebewohner wird nicht nur diese Perspektive verfolgt, sondern auch Rimbauds Sprache zugleich radikalisiert und versachlicht: In der Ausrichtung auf die urbane Epik geht das Bild des reisenden Autors Rimbaud in die Bestände des Mythischen ein, die Brecht aufruft, um die unter Druck stehende Erinnerung in einer urbanen Literatur des Vergessens zu modifizieren. Die in Dichtung und Rimbaud - Mythos vermittelte Ausrichtung auf die Figuren des Mörders, des Reisenden und des Dichters wird von Brecht durch einen generalisierenden Hinweis auf den Städtebewohner ersetzt, der über den Vitalismus zu einer neuen Verständigung zu gelangen sucht. So klagt Garga, der im Verlauf des Kampfes um eine unmögliche Freundschaft vom Provinzler zum Mörder und Nomaden wird, im Gespräch mit der Geliebten Jane : « [...] Ich habe ein Gefühl für dich. Es gibt ja keine Wörter! Aber wir werden uns wieder verständigen. Heut abend komme ich, schon diesen Abend!» (Brecht: Werke 1, 444). Die Suche nach einer neuen Sprache führt über den Körper und seine vitalen Ausdrucksformen, sie führt zu einer - ebenfalls bei Rimbaud angelegten - Wendung auf die in diesem Bruch aufgerufene Tradition der Epik und des Mythos, die auch Brechts Bezug zu und das Bild von Rimbaud organisieren. (Vgl. Rimbaud : Le bateau ivre, in : Seher - Briefe. Lettres du voyant. 50). Der Weg zu einer neuen Verständigung, an der Brecht seinen Gegenständen wie der Form nach arbeitet, verläuft nicht zuletzt über die Kritik an der Erinnerung und ist Teil von physiognomischen Entwürfen eines urbanen Menschen, der - wenn auch implizit Dichter geworden ist : ein Dichter, der Rimbauds in den « lettre du voyant » formulierte Erwartung einer objektiven Dichtung, die auf der Ausbildung des Sehnsinns beruht, radikalisiert zu einem ästhetisch - anthropologischen Entwurf und diesen in einem versachlichten Rekurs auf Rimbauds Themen und Bildwelten als eine Sprachpraxis gestaltet, die den Platz der entwerteten Erinnerung einnimmt. - Nicht als Dichtung, sondern als modernes Epos des urbanen Menschen, in dessen Sprache allein, sein neues Gesicht sichtbar wird. 


\section{Ein neuer Typus Mensch und das Problem der Verständigung}

4 Die von Brecht als Absage an Buchkultur und (familiäre) Genealogie geführte Kritik des Gedächtnisses ist also mehr als ein Reflex des Vitalismus. Dieser wird vielmehr in ein Lob des Vergessens eingearbeitet, wenn Brechts Abrechnung mit den Würdeformen der Traditionsbildung als Teil der Frage nach der Sprache und der Verfassung eines urbanen Menschen gelesen wird. Denn nicht nur die Literatur steht vor den Herausforderungen des urbanen Lebens : Mit den «großen Gebäude(n) der Stadt New York » bilde sich, so Brechts Diagnose, ein «neuer Typus Mensch » heraus. Ein Mensch, der, auch wenn er nicht so sei, wie der alte ihn sich gedacht habe, doch stets « wie ein Mensch» aussehen werde. (Brecht: Werke, Band 21, 208). Erst vor dem Hintergrund dieser Bemerkung, in der Erinnerung und zukünftiger Entwurf durch Denken und Anschauung ersetzt sind, erhält der Refrain im zweiten Dreigroschenfinale seine volle Bedeutung: "Denn wovon lebt der Mensch ? Indem er stündlich / Den Menschen peinigt, auszieht, anfällt, abwürgt und frißt / nur dadurch lebt der Mensch, daß er so gründlich / vergessen kann, daß er ein Mensch doch ist. » (Brecht : Werke, Bd.11, 144). Die Forderung dieser Verse, die nach den Bedingungen menschlicher Existenz fragen, kann keineswegs auf eine sozialkritische Intervention reduziert werden, in der Vergessen als eine Form von Enthumanisierung erschiene. In den vier Zeilen werden vielmehr verschiedene Begriffe des Menschen aufgerufen: Mit der Frage danach, wovon der Mensch lebe, ist auch die Frage gestellt: was ist, wer ist der Mensch? Die Perspektive des Idealismus wird in der ersten Antwort umgekehrt: der Mensch, der hier in Rede steht, ist nicht gut und wird in der dritten Zeile durch einen materialistisch - vitalen Elan gekennzeichnet. In dieser Umkehrung wird der hier befragte Mensch nicht zuletzt von seinen allegorischen Beschwörungen entlastet. Auf Ebene des Geistigen verfolgt er ein Vergessen des idealistischen Menschenbildes, wie es etwa im Expressionismus noch aufgerufen worden war. Zugleich wird Vergessen als eine Kritik der Medien und Zeiten der Erinnerung, als Absage an das Geistige formuliert. Diese, und darauf verweist das « doch », wird allerdings selbst als Literatur des Vergessens realisiert : deren Sprache kommt, nach dem Abschied von Allegorie und Metaphysik, materialistisch und sachlich daher. Nur in dieser Dimension, so scheint es, ist der Mensch doch Mensch. Brecht plädiert keineswegs einfach für ein Vergessen und einen Bruch mit der Vergangenheit. Vielmehr ist seine Literatur des Vergessens als eine Umarbeitung der Erinnerung $\mathrm{zu}$ lesen, die etwa in der Einleitung von « Hauspostille » als Angedenken dezidiert aufgerufenen wird, ohne dass jedoch in dem entsprechenden Abschnitt zu Erinnerndes positiv gestaltet würde. - Im Gegenteil : die Auslöschung steht auch hier im Mittelpunkt (Vgl.: Brecht: Werke 11, 40 und 107 f.). Das Angedenken ist in die Praxis einer Literarisierung eingezogen, in deren Sprache eine neue Kommunikation und die Überführung unmittelbarer Vitalität in eine symbolische Praxis der Sprache und der Aufführung, der Schriftsetzung und der Lektüre erhofft wird. In dieser Bewegung werden neue Strategien zur Aktivierung der Sinne unter den Verhältnissen der großen Städte ins Werk gesetzt, die nicht zu letzt auf dem Lob des Vergessens beruhen und Ritualisierungen an die Stelle positiver Inhalte der Erinnerung rücken. Diese Konstellation ist nicht nur in "Hauspostille » , sondern insbesondere auch in «Im Dickicht der Städte » zu verfolgen, in dem die Frage nach der angemessenen Form der Erinnerung ebenfalls auf den Mythos und die Formen 
der Epik verwiesen ist, die Brecht in seiner Bearbeitung für eine zeitgenössische Literatur gewinnen möchte.

Nicht zufällig ist Babylon der mythische Fluchtpunkt der in Chicago oder New York nicht mehr möglichen Verständigung: «Diese babilonische Verwirrung der Wörter / Kommt daher, weil sie die Sprache / Von Untergehenden sind [...].» (Brecht: Werke, Band 13, 356) Die hier unter der Überschrift « Die Städte« in eine mythische Tradition eingestellte Sprachverwirrung - auf die auch Brechts Drama reagiert - wird im Folgenden auf den Bruch der Genealogie und den Tod bezogen und durch den Bruch mit der Tradition ist sie explizit auf die Buchkultur ausgerichtet : auf die Frage, ob es in «Büchern vielleicht älterer Zeiten » nicht «Vorlage von Menschen " gegeben hätte, einen "alten, einfachen Plan ", nachdem sich zu richten möglich gewesen wäre, lässt Brecht mit Hinweis auf Sehsinn, Zahlensymbolik und Literaturpraxis antworten : «[...] die Pläne gab es / Aber sehet, sie waren beschrieben / Mit neuen Zeichen fünfmal darüber unlesbar / Fünfmal umgeändert die Vorlage nach unserm / Verkommenen Bildnis, [...]» (Ebd., 358). Die Erinnerung der Väter ist hier auf die Gegenwart verwiesen, kommunikativ oder gar Handlung anleitend ist sie nicht mehr. Die Frage nach Erinnerung und Gedächtnis, denen keine Legitimtions- oder Definitionsmacht für den neuen Typus Mensch mehr zufällt, wird von Brecht als ein Sprachproblem gestellt. Mit diesem ist sie Teil der Herausforderung des Menschen durch die Lebenswirklichkeit der großen Städte. Auch daher ist Brechts Beschreibung der Lebenswirklichkeit in den Städten mit der Kritik an der Erinnerung ebenso verschränkt, wie die den Städtern vorgeschlagenen Lebensstrategien mit den Modalitäten, in denen Erinnerung in einer Literatur des Vergessens umgearbeitet wird, die an den Platz des unbrauchbar gewordenen kulturellen Erbes rückt.

\section{Die Beschreibung mondialer Urbanität}

Wie beschreibt Brecht die Wirklichkeit des Urbanen, die zugleich einen Druck auf die Erinnerung ausübt und an der Modifikation des zu Erinnernden Anteil hat? Städte fallen mit einer Natur in eines, deren Bild nicht mehr als romantisches Gegenentwurf taugt und werden in eine mondiale Perspektive gesetzt. Der Mann « aus dem Netz des Verkehrs", dessen "Gesicht nicht wahrgenommen / Dessen geheimnisvolles Wesen unbeobachtet / Dessen Namen nicht deutlich gehört worden ist ", soll in " Anleitung an die Oberen » überschriebenen Versen durch eine Radioadresse geehrt werden: Dem unbekannten Mann soll gedacht werden mit «einer Arbeitsruhe der sämtlichen Menschen / Über den ganzen Planeten!»(Brecht: Werke, Bd. 11, 173). Ist hier also doch auf einen Akt des Angedenkens zu verweisen, wenn auch für einen Menschen, dessen Physiognomie ausgelöscht ist ? Mit der mondialen - wenn man so will : epischen - Perspektive wird hier zugleich ein Insistieren auf den Akt des Innehaltens eingeführt: Erst diese Ruhigstellung bietet die Möglichkeiten, neue Haltungen und Strategien zur Reaktivierung der Sinne in Anschlag zu bringen, die insbesondere der Umarbeitung einer - auch hier aufgerufenen - Erinnerung dienen. Sie verleihen zugleich dem neuen Typus Mensch wie der urbanen Literatur Brechts Gestalt. Metaphysische Transzendenz und retrograde Traditionsbildung sind für diesen und in dieser - wie auch das als Referenzpunkt nicht mehr taugende Mittelmeerbecken durch die präsentische Ordnung planetarer Räume ersetzt. Diese werden von Brecht insbesondere mit Blick auf die Städte Nordamerikas, das atlantische Meer und die 
Sphäre des Kosmos gestaltet. - Etwa in den folgenden zum Anhang der « Hauspostille » gehörenden Versen aus « Vom armen BB » :

Wir sind gesessen ein leichtes Geschlecht

In Häusern, die für unzerstörbar galten

(so haben wir gebaut die langen Gehäuse des Eilands Manhattan

Und die dünnen Antennen, die das Atlantische Meer unterhalten).

(Brecht : Werke, Bd. 11,120)

7 Der Mensch hat in dieser technisierten Kommunikation, die auf die Ausgestaltung eines mondialen Sprachraums zielt, keinen Platz mehr; ebenso wenig wie eine metaphysische Aufladung der Atmosphäre, die an anderer Stelle vielmehr in eine knappe Landschaftsbeschreibung und Metaphorik eingeholt wird, die das Gesicht des gegenwärtigen Menschen in den Blick nimmt. Wie ist die Lebenswirklichkeit in einer solchen umfassenden Urbanität gekennzeichnet? Neben Sprachverwirrung und einer nicht möglichen Verständigung stehen als Elemente urbanen Lebens im « Dickicht der Städte » : Chaos, Kälte und Nichts. Das Chaos sei, so der Chicago Richtung New York verlassende Garga, aufgebraucht und er kommentiert lakonisch: "Es war die beste Zeit. » (Brecht : Werke, Bd. 1, 497). Doch New York ist nicht weniger kalt, der Winter ist die einzige Jahreszeit des urbanen Lebens : «Frühjahr, Sommer und Herbst - wie ich euch sagte, sind den Städten ein Nichts, aber der Winter ist merkbar. » (Brecht : Werke, Band G 3, 389). Das Nichts steht in den Städten neben der kalten Jahreszeit, die - wie hier in den Versen von " Über den Winter » - von ihrer allegorischen Verklärungen in der Dichtung entlastet wird : Kälte verweist auf den Tod, auf nichts sonst. Nicht nur die Jahreszeiten werden im Blick Brechts auf die Wirklichkeit des urbanen Lebens und auf die in diesem in Anschlag zu bringenden Strategien auf ein Nichts reduziert: Die selbe Diagnose gilt auch für die Menschen, deren Porträts in «Dickicht der Städte » gezeichnet werden: "Mae Garga, geboren 1872 in den Südstaaten, verschollen im Oktober vor drei Jahren, sie ist sogar aus der Erinnerung verschollen, sie hat kein Gesicht mehr. Es ist ihr abgefallen, wie ein gelbes Blatt. » (Brecht : Werke Bd.1, 489). Das Nichts gerät zum Zentrum des urbanen Lebens und ist letztlich der Gegenstand von Brechts literarischer Gestaltungsanstrengung, dem auch die Kritik der Erinnerung geschuldet wird :

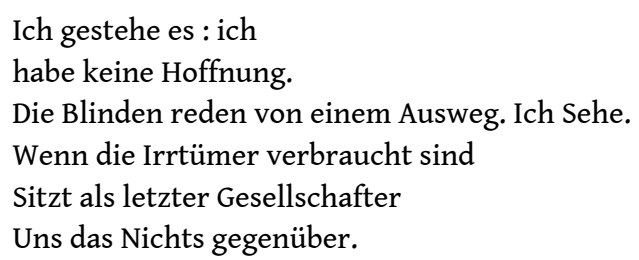

(Brecht : Werke, Bd.13, 189)

8 In diesen Zeilen aus «Den Nachgeborenen » ist kein Fortschrittsprojekt aufgerufen, gibt es kein als Erinnerung zu akkumulierendes Erbe mehr, auf das dieses aufbauen könnte : statt dessen ein aufzubrauchender Vorrat an Irrtümern, durch deren Abnahme der Blick auf eine Leere freigegeben wird, die den Platz des akkumulierten Wissens und einer auf dessen Erinnerung basierenden Identität einnimmt. Zugleich wird jedoch hier auf die sinnliche Dimension der Wahrnehmung verwiesen und die Dimension des Subjekts unterstrichen. Das literarische Ich, das seine Kontur auch aus der Widmung des Gedichts an die «Nachgeborenen » erhält, wird mit dem Nichts konfrontiert und diese Konstellation beschreibt die Aufgabe, der sich Literatur zu stellen hat ; zumindest dann, wenn Leben und Verständigung der Menschen unter den Verhältnissen des Urbanen gewährleistet werden sollen. Neben den Bildern, in denen das urbane Leben 
und die Herausforderungen beschrieben werden, auf die Literatur zu reagieren hat, um zur Darstellung fähig zu bleiben, finden sich hier bereits Perspektiven einer möglichen Antwort: Brechts Strategien zur Reaktivierung der Sinnes, die aus seinen Porträts Gargas oder des armen B.B. gewonnen werden können, sind auf Rimbauds Ausschweifung aller Sinne bezogen, in denen dem Visuellen eine besondere Bedeutung zukommt. Welche Strategien haben an diesen Lebenspraktiken Anteil, die als Teil der Umarbeitung von Erinnerung zu lesen sind? An erkennendes Denken und an einen gegenwärtigen Genuß ist zunächst zu denken, die aus Rimbauds Vitalismus und Visualismus gespeist werden, an eine Aufmerksamkeit für archaische Muster in der Verfassung des urbanen Lebens und epischer Traditionen in den literarischen Formen ihrer Beschreibung ebenso, wie an eine neue Verbindung von Natur und Kultur in der Ordnung der großen Städte, deren Bildwelt ebenfalls an den Platz der Erinnerung gerückt wird. Eine besondere Bedeutung gewinnt schließlich die Dimension der Handlung und eine dieser verbundenen Sprachlichkeit. So wird 1926 die Vergänglichkeit der Stadt unter der Überschrift «Behauptung " hervorgehoben und zugleich mit einer Dimension der Tätigkeit verbunden. Diese kann so neben das Schreiben, das Sprechens und die Lektüre gesetzt werden, die in den Anweisungen zum Gebrauch der « Hauspostille » unterstrichen sind :

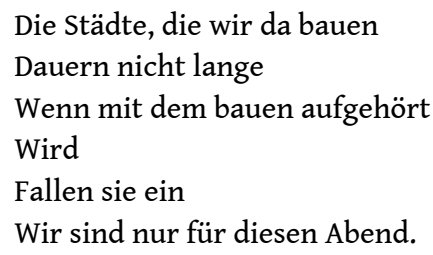

(Brecht: Werke, Bd.13, 336)

9 Auch im «Dickicht» wird der Akzent auf die Dimension der Handlung gelegt : Kann doch - Paul Ricoeur folgend - neben Interaktion, Kooperation und Wettbewerb auch der Kampf eine Formen der Handlung sein. (Ricoeur : Zeit und Erzählung I, 91) Auch indem in der Literatur Brechts diese Dimension betont wird, ist der Akzent auf ein Vorverständnis der Welt des Handelns gesetzt, in deren Präfiguration die Fabelkomposition - Ricoeurs Modell der dreifachen Mimesis entsprechend - verwurzelt ist. Dieser Verschiebung von der Literatur als Werk hin zu einer Literarizität der Welt als Handlung, in der das Vorverständnis der Fiktionen des Als-ob verankert ist, entspricht zugleich eine Aufwertung der Rezeption und der Refiguration in der dritten Stufe der Mimesis. Auch diese wird von Brecht sowohl den Gegenständen seiner Literatur wie ihrer Konzeption nach unterstrichen. Zwischen beiden Bereichen steht die Fabel - und mit ihr eine in ihr ungesicherte oder nicht mehr zugängliche Erinnerung - nur noch als eine Stufe der Vermittlung: ihre Abwertung ist Teil einer von Brecht angestrebten Objektivierung der Dichtung, die zugleich eine, hier eminent literarische Wandlung der Erinnerung ist : ein Bezug zum Mythos wird aufgewertet, der im Wortsinn der aristotelischen Poetik als eine «Zusammensetzung der Handlungen » (Ricoeur : Zeit und Erzählung I, 104) verstanden wird und Gegenstand sprachlicher Berarbeitung wird.

Welche Bedeutung haben Mythos und Bezüge zu epischen Formen der antiken Literatur für Brechts Transformation der Erinnerung in einer Literatur des Vergessens, die auf eine Aufwertung der (Sprach-) Praxis in Strategien zur Reaktivierung der Sinne setzt? Um diese Frage zu beantworten, scheint es notwendig, genauer herauszuarbeiten, in welcher Weise die Arbeit an einer neuen Epik der Städte, über die 
eingerissenen Brücken des (neu-) humanistischen Erbes hinweg, auf die Mythen bezogen ist, die seit je - und so scheint es gerade auch in der Kritik der Erinnerung die Frage nach dem Bild des Menschen grundieren. Am Ende von «Das Dickicht der Städte ", dieser dem Boxkampf, dem Verbrechen und der Reise folgenden Odyssee des urbanen Menschen aus seiner Erinnerung in die Sprache des Vergessens, ist Gargas Gesicht verwandelt: Hatte der Provinzler zu Beginn noch seine Erinnerung an die Landschaften der Kindheit, als Bild vor sich, das in seinem Antlitz zu lesen war, ist am Ende des Kampfes diese Erinnerung ebenso aufgebraucht, wie das Chaos und das Nichts in dem die familiären Bindungen des Städters aufgelöst sind. Es bleiben : Gargas Gesicht - ein durchsichtiger Bernsteins, in dem Tierleichen eingeschlossen sind -, das den Tod des feindlichen Freunds ankündigende "Geheul der Lyncher» (Brecht: Werke, Bd.1, 480), die bald nach diesem Gespräch die Szene eines Eisenbahnerzelts in der Peripherie der großen Stadt betreten und eine Stille, in der die neue Sprache als Gespräch zwischen Shlink und Garga zu studieren ist : « Das immerwährende Geräusch Chicagos hat aufgehört. Siebenmal drei Tage sind die Himmel verblaßt, und die Luft ist graublau geworden wie Grog. Jetzt ist die Stille da, die nichts verbirgt. » (Ebd., 490). - So eröffnet Shlink die Szene. In diesem Gespräche der beiden Kämpfer im " Dickicht der Städte », einem Moment größter Nähe und Distanz zugleich, ist auf die angemessene Fassung des zu Erinnernden hingewiesen: Leben und Gedächtnis fallen auseinander, die Ausrichtung auf die Finalität des Daseins wird zugunsten eines als Kampf vorgestellten Handlungsmodells des Lebens ebenso aufgegeben, wie die Möglichkeit, dieses erinnernd zu fassen: "Die Etappen des Lebens sind nicht die der Erinnerung. Der Schluß ist nicht das Ziel, die letzte Episode nicht wichtiger als irgendeine andere [...]. » (Ebd.). Nicht nur das Individuum fällt in der urbanen Wandlung des Menschen und seiner Sinne aus der Erinnerung aus, das Leben selbst geht dieser verloren. Mit dieser Wandlung, gehen Umwertungen zentraler Elemente des in die Krise geratenen humanistischen Menschenbildes einher : Feindschaft allein ermöglicht « Fühlung », die nicht mehr nur auf einen Menschen, sondern auf den Planeten bezogen wird, in dessen Bild die Physiognomie des urbanen Menschen aufgelöst wird. Der über vierzig Jahre einsam gebliebene Shlink verfalle, so Garga, an seinem Ende « der schwarzen Sucht des Planeten, Fühlung zu bekommen.» (Ebd.). - Durch eine Feindschaft, in der die beiden kämpfenden Männer « Kameraden einer metaphysischen Aktion » werden, an deren Ende der junge Mann und das «Prinzip Stadt» den Sieg über den Alten davonträgt (Ebd.). Zugleich mit dem Hinweis auf die Übersetzung einer ausfallenden Erinnerung kann der sprachliche Stil dieses Dialogs als Beispiel für die neue Form der möglichen Verständigung gelesen werden. Diese wird hier als Physiognomie der Landschaft eines Menschen entworfen, der sein auf Erinnerung und Genealogie basierendes Gesicht verloren hat. - Ein Bild von epischer Dimension, die grade durch die Umkehrung von Metaphysik und Idealismus erschlossen wird, die eben auch durch die Aufgabe der Erinnerung zugunsten eines sich in den Landschaft der Städte absetzenden Mythos eröffnet wird. Doch wie sind Petrifikation, Mord und Schweigen mit dem Modus verbunden, in dem in einer Literatur des Vergesssens der Bezug auf den Mythos als Ersatz der getilgten Erinnerung organisiert wird? Ein Blick in Brechts Bearbeitung des Sirenenkapitels aus Homers Odyssee kann hier weiterhelfen. 


\section{Der Provinzler Odysseus und das Schweigen der Sirenen}

11 Mit dem Sirenenstoff wird von Brecht nicht nur ein Verweis auf die Reise, sondern eine prominente Allegorie der Kunst aufgerufen und im Zeichen des Urbanen bearbeitet: Odysseus wird in dieser Bearbeitung nicht als der Listige, sondern als "vorsichtige(r) Provinzler« vorgestellt. (Brecht: Gesammelte Werke 11, Prosa I, 207)5. Der Gesang der Menschen fressenden Sirenen gerät so zum Rauschen der Großstadt : dass Odysseus gefesselt - ihm nicht erliegen kann, die anderen Irrfahrer - Wachs in den Ohren - ihn zugunsten der Stille ihrer künstlich herbeigeführten Taubheit nicht vernehmen, führt Brecht zur Frage, ob der Gesang der Sirenen überhaupt zu vernehmen war ${ }^{6}$. Die Erinnerung an den Gesang der Sirenen, die nur durch das Zeugnis des Odysseus verbürgt ist, wird in der Bearbeitung bezweifelt. Der kunstvolle Gesang ist in dieser Variante durch ein kraftvolles Schimpfen ersetzt. Nachdem Brecht die Episode des mörderischen «Kunstgenuß» in der Variante von Homers Odysseus berichtet hat, fragt er :

[...] Sollte ich der erste sein, dem Bedenken aufsteigen? Ich sage mir nämlich so : alles gut, aber wer - außer Odysseus - sagt, daß die Sirenen wirklich sangen, angesichts de angebundenen Mannes? Sollten diese machtvollen und gewandten Weiber ihre Kunst wirklich an Leute verschwendet haben, die keine Bewegungsfreiheit besaßen? Ist das das Wesen der Kunst? Da möchte ich doch eher annehmen, die von den Ruderern wahrgenommenen geblähten Hälse schimpften aus voller Kraft auf den verdammten, vorsichtigen Provinzler, und unser Held vollführte seine (ebenfalls bezeugten) Windungen, weil er sich doch noch zu guter Letzt genierte ! (Ebd.).

Der Zweifel Brechts setzt an der verbürgten Überlieferung an: der kollektiven Erinnerung, für die es keine Gewähr gibt. Die Bearbeitung der Sage, die berichtet und in der Bearbeitung modifiziert wird, ist eine literarische Substitution des in der Auslegung vergessenen: habe das Erinnerte so wie behauptet - zumindest im Zweifel des erkennenden Denkens, den Brecht hier vorführt - doch nicht stattgefunden. Der Akt des Denkens und der Bearbeitung wird in Spannung zur tradierten Überlieferung, die als «bekanntlich » eingeführt wird, selbst präsentiert. Er wird so zu einem Teil des neuen Sirenenstoffes. Worin besteht die spezifische urbane Dimension dieser Bearbeitung? Zunächst bereits in dieser Transparenz, die den Prozess- und Handlungscharakter der Bearbeitung einsichtig macht, dann mit dieser auch in der sprachlichen Form: Der Sirenenmythos wird hier in einer sachlichen, zur Lakonie tendierenden Sprache berichtet. In dieser ist die überlieferte Episode von allen Allegorien entlastet, die sich in ihrer Tradierung abgelagert hatten. Weiter: Das Verhalten des Odysseus kennzeichne ihn, so Brecht, als Provinzler. Als Städter wäre Odysseus, sich seiner Feigheit genierend, in der Lage gewesen, den mörderischen Gesang der Sirenen auszuhalten. Auf die Städte verweist mit der Mordlust der Schönen auch die Stille, von der die Männer umgeben sind, deren Gehörsinn mit Wachs verschlossen ist. Sind doch die Irrfahrer einer stets aufs neue aufgeschobenen Heimkehr in dieser durch eine Armierung der Sinne erzeugten Stille dennoch - oder gerade deshalb - in der Lage, dem modernen Sirenengesang der Städte zu lauschen. Das Wachs dient als Filter vor der Membran des Ohres, es ist Teil einer notwendigen Modifikation der in einer urbanen Lebenssituation zugleich aufgewerteten Sinne. Der Gesang der städtischen Sirenen ist nun entweder stark gefiltert zu hören oder aber nur 
in der Einbildung zu imaginieren. Diese wird am Platz der Stille zur Sprache gebracht; sei es, dass sie phantasmatisch eine Erinnerung substituiert, sei es, dass sie einen gefilterten Gesang ergänzt, der gleichsam aus der Ferne auf das Ohr trifft. Der Gesang, der hier zu vernehmen ist, kann jedoch auch als Ergänzung zu oder als Bericht von einer Erinnerung gelten, der nicht geglaubt wird, die vergessen werden kann und durch eine andere Geschichte zu ersetzen ist : Die Erinnerung selbst ist eine Erfindung. Aber weniger über den Gesang der Sirenen wird hier von Brecht spekuliert, als über den Status der Kunst, für die dieser allegorisch geworden ist. Der Gesang ist durch das Schimpfen ersetzt: in ihm wird die neue Sprache begründet, die Brecht für eine Stadtepik sucht, deren Kommunikationssituation in dieser Bearbeitung, die nicht weniger als die Poetik einer Literatur des Vergessens en miniature enthält, festgehalten wird. Das Schimpfen der Sirenen steht mit der babylonischen Sprachverwirrung am mythischen Ausgangspunkt einer neuen Sprache der großen Städte, die ihren Endpunkt in der Unterhaltung der in "Vom armen B.B. « angeführten Antennen mit dem atlantischen Meer findet. Die Sirenen, dem Provinzler ihre ebenso verführerischen wie mörderischen Künste verweigernd, singen nicht mehr - es sei denn in der Einbildungskraft, vermittelt durch spezifisch armierte Sinne oder durch die bearbeitete Erinnerung, die gerade im Bruch mit dem humanistischen Erbe und nicht mehr länger haltbaren kulturellen Gewissheiten ansetzt. Nicht nur die Bearbeitung der Sirenenepisode rücken die Sinne in das Zentrum von Brechts urbaner Literatur. Der Anpassungsprozess, dem etwa Garaga unterworfen ist, zielt ebenfalls auf die Sinne der Städtebewohner: diese werden zugleich umgebaut, um unter den Bedingungen des Urbanen tauglich zu sein und sind - wie die Auszeichnung von Rimbauds Vitalismus auch in Brechts versachlichter Variante zeigt - zugleich Teil einer Strategie zur Reaktivierung der Sinne. Diese ist nachgerade das Anliegen der Literatur und von Brechts Arbeit an einer ihrer ästhetisch - anthropologischen Dimension angemessenen Sprache der Verständigung. In den Städten ist der Kontakt, den die Sinne zur Umwelt herstellen, nicht mehr unmittelbar, sondern durch Armierung vermittelt : Dient diese nun - wie das Wachs in der urbanen Variante der Sirnenepisode - der Abschwächung oder aber der Steigerung der Sensation, die zum Gegenstand der Erinnerung oder des Vergessens wird. Auch das Gedächtnis findet in der Schrift seine Armierung, die jedoch nicht unbedingt auf akkumulierte Erinnerung zielen muß, sondern auch der ars oblivionis verpflichtet sein kann. Wie die mittelmeerische Welt des Südens durch das atlantische Meer und eine kosmische Atmosphäre ersetzt ist, taugt in dieser auch Odysseus, anders als in den zahllosen Bearbeitungen des Neuhumanismus, nicht mehr zum Vorbild eines urbanen Menschen ${ }^{7}$. An Stelle der individuellen Erinnerung, die durch ein Lob des Vergessens ersetzt wird, um in den Städten lebensfähig zu bleiben, und an Stelle der kollektiven Erinnerung, die unter dem Druck der urbanen Gesellschaft zugleich mit Metaphysik und Idealismus aufgegeben wird, ruft Brecht die Erinnerung des Mythos als Teil der Arbeit an einer neuen, auf den Menschen zielenden Sprache auf. Doch auch diese ist gebrochen und wird von ihm in der Bearbeitung als Teil eines neuen urbanen Epos an die Stelle der Erinnerung und der Tradierung der Antiken gesetzt. Statt der individuellen Erinnerung eines Menschen, der in den Städten in das Vergessen der Auslöschungs- und Todesphantasmen zu fallen droht, wenn er sie nicht für das Vergessen optiert, wird ein mythisches Gedächtnis aufgerufen, das an Jahreszeiten und Landschaften gebunden ist, die Brecht in seine urbanen Szenerien integriert : « Von den Wäldern nehme ich das Schweigen mit. Aber von den Wiesen die Aufwärtsbewegung und die Gelassenheit von den Äkkern, die unter der Sichel wachsen 
für die Novembernächte.» (Brecht: Werke, Bd. 11, 29). Eine Brücke zu dieser Ebene eines Gedächtnisse der "Natur» - hier in «Eisenbahnfahrt, 19. Psalm» (1920) entworfen - ist der von Rimbaud gewonnene Vitalismus reiner Gegenwärtigkeit. Dieser wird zulasten der Vergangenheit und der Zukunft, der Erinnerung und der Zukunft forciert und eröffnet gemeinsam mit der Auszeichnung visueller Bildmedien einen gebrochenen Bezug zum epischen Erbe ${ }^{8}$. Nicht zuletzt solche Landschaften des Vergessens, in denen das Gesicht der individualisierten Menschen aufgelöst wird, tragen dazu bei, eine neue Epik der großen Städte zu konstituieren. Brecht reagiert auf die Herausforderung der Literatur und des Menschen durch den Einzug in die großen Städte mit einer Episierung des Dramas und neuen Darstellungsformen des Ichs. - Für beide, die nicht zuletzt auch eine neue Sprache verlangen, gewinnt die Frage nach Funktion und Ausdrucksweise des zu Erinnernden eine zentrale Rolle. Ihre Antwort wird in den Geschichten des Mythos gesucht, die Brecht in den mondialen Raum des Urbanen und in die Frage nach der Bildungsfunktion der Sprache überträgt.

Welche Gestalt nimmt die Erbform des Odysseus in diesem « Dickicht der Städte« an ? Als Protoyp des Städters setzt sich nicht zuletzt Brecht selbst in Szene. Etwa in der Variante, die er 1927 in den fotografischen Portätstudien Konrad Reßlers gibt: hartleibig in einem schwarzen Ledermantel, mit Rauch- und Schreibzeug ausgestattet ${ }^{9}$. Aus dem nicht mehr listigen, vielmehr provinziellen Odysseus ist hier ein durch die Armierung der Sinne auf Distanz setzender Autor und in der Gestalt des mythischen Helden Garga ein Mörder geworden; ein Städter, der seine Freundschaft und Lebensfähigkeit stiftende, wenngleich mörderische Handlung zugleich mit einem medial gebunden Akt des Vergessens verbindet. In diesem ist die Möglichkeit einer Zukunft wenn auch um den Preis der Negation des - so oder so endlichen - Lebens eröffnet, die nicht mehr auf der Erinnerung, sondern auf einer Literatur des Vergessens und ihrem Handlungspotential beruht. Am Ende des siebten Bildes von «Im Dickicht der Städte« wird die Zeitstruktur des Dramas aufgebrochen. Ist diese - wie auch Brechts urbane Lyrik - von einem stark präsentischen Charakter geprägt, der nur in der Erweiterung des jeweiligen Tages durch Gestern und Morgen ausgedehnt wird, schließt der Wendepunkt der dramatischen Handlung mit einem ausgreifenden Futur : Die Wendung auf diese Zeitform ist hier jedoch auch mit einem dezidierten Lob des Vergessens verbunden und dieses mit der Medialität der Schrift :

Ich schreibe diesen Brief, und ich stecke dieses Dokument hier in meine Tasche, damit ich alles vergessen kann. Und nach drei Jahren, denn so langen werden sie mich einsperren, werde ich, acht Tage vor meiner Entlassung, dieses Dokument der Zeitung übergeben, damit dieser Mann aus dieser Stadt ausgetilgt ist und aus meinen Augen verschwunden, wenn ich sie wieder betrete. Aber für ihn wird der Tag meiner Entlassung durch das Geheul der Lyncher angezeigt werden. (Brecht: Werke, Bd. 1, 480-481).

Indem die Futurformen in die Gegenwart der Städte zurückgeworfen werden, ist eine unbedingte Utopielosigkeit eingeführt, die dem Bruch mit der retrograden Bindung korrespondiert. An die Stelle linearer Zeitspannung tritt ein Zusammenhang von vitaler Präsenz, Visualismus und Verräumlichung, der Brechts urbane Epik bestimmt. Er bildet den Hintergrund der Entfaltung einer ars oblivionis, in der das Lob des Vergessens als Umarbeitung der Erinnerung und als ästhetisch-anthropologisches Handlungspotential der Literatur entfaltet wird. Gerade in dieser Konstellation wird die von Brecht zulasten von idealistischer und metaphysische Aufladung der Literatur geforderte Versachlichung betrieben, die hier auch durch die Auszeichnung von Brief, 
Dokument und Zeitung unterstrichen wird; wenn zugleich auch eine beinahe alttestamentarische Verve zu verzeichnen ist, die auf die Ritualsierungen dieser Sprachpraktiken in ihrer Aufführung hinweist. Mit diesen Referenzen einer gleichwohl sachlichen Spracherneuerung kann auch das Geheul der Lyncher als Indiz einer Erneuerung der Literatur gelesen werden. Es tritt mit dem Schimpfen der Sirenen an die Stelle der hohen Kunst und einer Sprache, die nicht mehr in der Lage ist, die Wirklichkeit der Städte zu erfassen. Das Entlastung versprechende Vergessen, mit dem die Drohung einer angekündigten Vernichtung verbunden ist, wird als Teil einer Medialisierung vorgestellt, der zugleich eine Handlung anleitende Dimension zukommt. Der Brief - im Sinne Freuds ein «materialisiertes Stück des Erinnerungsapparates " - wird die Voraussetzung des Vergessens und Agens einer vernichtenden Handlung. (Freud: Wunderblock, 365). Diese wird zuerst von Brecht selbst in seiner Arbeit an einer Literatur und an Lebensstrategien der Städtebewohner betrieben. In ihrer Summe sind diese nichts anderes als ein solcher Brief, der aus der Tiefe des Mythos in den urbanen Raum zugestellt wird. - Als Hinweis auf eine neue Sprache, die in ihrer Praxis nicht weniger sein will als das Bild eines urbanen, sich im Vergessen tätig erfindenden Menschen.

\section{Die Texte Brechts werden nach den folgenden Ausgaben zitiert :}

Bertolt Brecht : Werke. Große kommentierte Berliner und Frankfurter Ausgabe. Hrg. v. Werner Hecht, Jan Knopf, Werner Mittenzwei, Klaus Detlef Müller. Frankfurt am Main : Suhrkamp, Berlin und Weimar : Aufbau $1988 \mathrm{f}$.

Bertolt Brecht : Gesammelte Werke, Band 11, Prosa I, Frankfurt am Main : Suhrkamp 1967.

\section{Weitere Literatur :}

Henri Bergson : Materie und Gedächtnis, Hamburg : Meiner 1991. Sigmund Freud : Notiz über den « Wunderblock» (1925 [1924]), in : Freud Studienausgabe Band III, Psychologie des Unbewußten, hrg. v. Alexander Micherlich u.a., Frankfurt am Main : Fischer 1994.

Helmut Lethen : Verhaltenslehren der Kälte. Lebensversuche zwischen den Kriegen, Frankfurt am Main : Suhrkamp 1994.

Klaus-Detlef Müller (Hg.) : Bertolt Brecht. Epoche - Werk - Wirkung, München : Beck 1985 (mit einer ausführlichen Bibliografie).

Paul Ricoeur : La mémoire, l'histoire, l'oubli, Paris : Éditions du Seuil 2000.

Paul Ricoeur : Zeit und Erzählung. Bd. 1. Zeit und historisch Erzählung, München : Fink 1988.

Arthur Rimbaud : Seher - Briefe. Lettres du voyant, Übersetzt und hrg. v. Werner von Koppenfels. 
Mainz : Dieterich'sche Verlagsbuchhandlung 1990.

Renate Schlesier : « Das Schweigen der Sirenen«, in : Christoph Wulf / Dietmar Kamper (Hg.) : Logik und Leidenschaft. Erträge Historischer Anthropologie. Berlin : Reimer 2002, 1089-1097. Harald Weinrich : Léthé : Art et critique de l'oubli. Traduit de l'allemand par Diane Meun, Paris : Fayard 1999.

\section{NOTES}

1. Ich zitiere aus der im Literaturverzeichnis angegebenen Frankfurter und Berliner Ausgabe der Werke Brechts.

2. Hier wäre etwa an Ernst Robert Curtius «Europäische Literatur und Lateinisches Mittelalter zu denken oder auch an die Bemühungen Aby Warburgs und seiner Kulturwissenschaftlichen Bibliothek. Vgl. auch Christoph König und Eberhard Lämmert (Hg.) : Konkurrenten in der Fakultät. Kultur, Wissen und Universität um 1900. Frankfurt am Main : Fischer 1999.

3. Dies geschieht auch mit Blick auf die deutsch-französischen Geistesbeziehungen der Zwischenkriegszeit. Vgl. v. Verf. : «Literarische Annexion? Borchardts Übersetzung zwischen Politik und Phantasma. In : Kai Kauffmann (Hg.) : Dichterische Politik. Studien zu Rudolf Borchardt. Publikation zur Zeitschrift für Germanistik 4, Bern 2002, 183-204.

4. $\mathrm{Zu}$ der hier insbesondere relevanten «zweiten unzeitgemässen Bemerkung« Nietzsches und zur Bedeutung der Topologie in dessen Geschichtskritik vgl. v. Verf. : Sprachen der Freundschaft. Rudolf Borchardt und die Arbeit am ästhetischen Menschen. München : Fink 2003.

5. Ich zitiere aus den im Literaturverzeichnis angeführten Gesammelten Werken Brechts.

6. Dies hat zuerst Renate Schlesier unterstrichen, der ich hier folge. Vgl. : Literaturverzeichnis.

7. Eine andere Position könnte in Joyces « Ulysses« studiert werden. Vgl. : Hugh Kenner : Ulysses. Frankfurt am Main : Suhrkamp 1982.

8. Vgl. das Nachwort von Koetzle und die Aufnahmen in: Bertolt Brecht beim Photographen. Porträtstudien von Konrad Reßler. Hrsg. und mit einem Nachwort versehen v. Michael Koetzle. Berlin : Nishen, 1989.

9. Vgl. Anmerkung 8.

\section{RÉSUMÉS}

Unter den Bedingungen eines urbanen Lebens, dessen Anamnese am Beginn von Brechts literarischer Arbeit steht, wird die Verbindung zwischen abstrahierter Erinnerung und auf dieser gründenden Fortschritt aufgebrochen. Der Modus des Gedächtnisses ist statt dessen mit Prozessen und Praktiken der Literarisierung verbunden. Diesen fällt in der notwendig werdenden Anpassung des Menschen an die Bedingungen einer neuen urbanen Qualität ein ästhetisch - anthropologisches Gestaltungspotential zu, das nicht zuletzt in der Literatur selbst im Verhältnis zu einer ihre Definitionsmacht einbüßenden Erinnerung diskutiert wird. In Brechts Arbeiten aus den zwanziger Jahren des letzten Jahrhunderts kann eine Literatur des Vergessens studiert werden, die Teil seiner Bemühung um eine urbane Sprache und um neue Lebenstrategien für Städtebwohner ist. Die Auszeichnung der Erinnerung wird in diesen nicht 
einfach negiert, sondern unter Auszeichnung der Handlungsdimension in eine sprachliche Praxis umgearbeitet, die einem Lob des Vergessens den Vorrang vor der Akkumulation des Vergangenen gibt.

Les modalités de la vie urbaine, dont l'évocation sous forme d'anamnèse constitue les prémices de l'œuvre littéraire de Brecht, conduisent à une rupture du lien entre le souvenir abstrait et le progrès qui en procède. Le travail de mémoire est dès lors lié à des processus et des pratiques de littérarisation qui développent les possibilités d'une approche esthétique et anthropologique reflétant la nécessaire adaptation de l'homme aux conditions d'une nouvelle qualité urbaine. Cette approche est l'enjeu d'un discours littéraire aux prises avec le souvenir qui a lui-même perdu son pouvoir de définition. On se propose d'étudier ici, dans les travaux de Brecht datant des années vingt du siècle dernier, une littérature de l'oubli qui participe d'une tentative de développer une langue urbaine et de nouvelles stratégies de vie adaptées aux citadins. La négation du primat du souvenir, auquel se substitue la dimension de l'action, permet de le transformer en une pratique linguistique où l'accumulation du passé cède le pas à un éloge de l'oubli.

\section{AUTEUR}

\section{FRANCK HOFMANN}

Université Charles-de-Gaulle - Lille 3 